\title{
Experimental validation of a numerical simulation on a ball- screw system by 3D photoelasticity
}

\author{
Y. Barranger ${ }^{1, a}$, R. Bertolaso ${ }^{2}$, P. Doumalin ${ }^{1}$, J.C. Dupré ${ }^{1}$, A. Germaneau ${ }^{1}$, and M. Cheikh ${ }^{3}$ \\ ${ }^{1}$ Intitut Pprime UPR 3346, CNRS - Université de Poitiers - ENSMA, 86962 Futuroscope \\ Chasseneuil Cedex, France \\ ${ }^{2}$ Mines d'Albi, CROMeP, 81013 Albi Cedex 09, France \\ ${ }^{3}$ Université de Toulouse II, IUT de Figeac, 46100 Figeac, France
}

\begin{abstract}
The Trimmable Horizontal Stabilizer Actuator (THSA system) equips the whole airbus line. One component of this system is a ball-screw system on which spalling problems appear on the balls. This phenomenon is mostly due to local high pressures and reduces the service life of the system. 3D numerical simulations are usually used to tackle this kind of problems but are subjected to assumptions. As the aim of the project is to build a numerical model able to predict pressure distribution, these assumptions need to be experimentally assessed to be perfectly relevant of the real load distribution in the ball screw system. Due to the 3D geometry of the specimen, a 3D measurement technique, Scattered Light Photoelasticity (SLP), has been chosen to perform experimental measurements,. Because of complexity of the geometry, the study is divided in three steps; the present paper is dealing with the second one where a demonstrator ball-screw system is manufactured in casted epoxy to perform the SLP. This technique gives information on 3D stress fields inside the epoxy specimen from the analysis of photoelastic fringes. They are compared to numerical ones and indicate whether numerical boundary conditions are relevant of the experimental ball-screw system behaviour.
\end{abstract}

\section{Introduction}

The THSA (Trimmable Horizontal Stabilizer Actuator) system is located at the back of aircrafts on the whole Airbus line (figure 1-a). Its role is to insure the guidance of the horizontal deflector by means of a ball screw system which assures the movement of a nut along the screw thanks to a recirculation system of balls (figure 1-b).

The problem encountered on this ball-screw system is a premature spalling phenomenon on balls which reduces significantly the service life of the whole system (figure 2). This spalling of balls is induced by contact at the interface between balls and the furrow of the screw, as well as the interface between the balls and the furrow of the nut. The contact pressure distribution is then an important parameter to study for this kind of mechanical system.

\footnotetext{
a e-mail : yoann.barranger@univ-poitiers.fr
} 
Spalling problems are generally induced by local high pressures. It is then necessary to know their maximum values at the vicinity of the contact zone. Hertz theory [1] gives an analytical approximation of the contact pressure distribution $(P(r))$ in the case of two spherical systems interacting with each other:

$$
P(r)=\frac{3 F_{n}}{2 \pi a^{2}} \sqrt{1-\frac{r^{2}}{a^{2}}}
$$

where $a$ is the radius of contact, $F_{n}$ the normal force and $r$ the radial polar coordinate. This analytical formulation makes the assumption that the contact region has to be small in comparison with the smallest radius of curvature of the contact bodies.

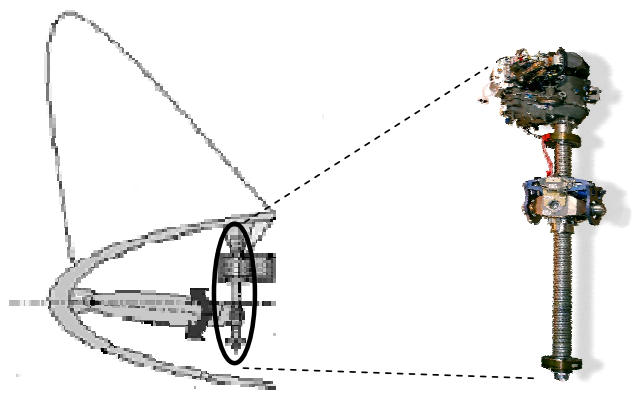

(a)

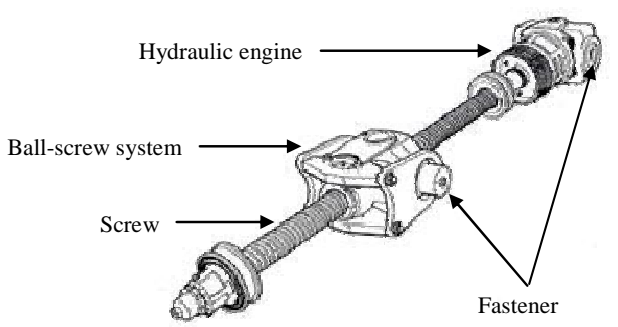

(b)

Fig. 1. (a) THSA system localisation on aircrafts and (b) main components

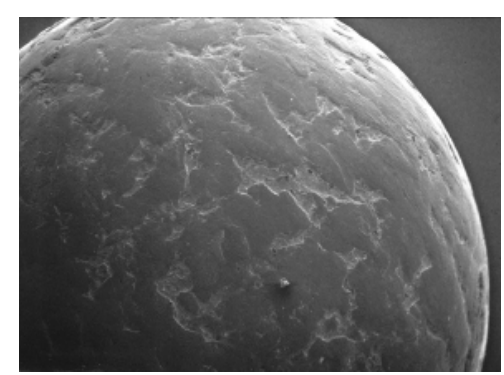

Fig. 2. Spalling phenomenon on the balls

In order to study these assumptions, this kind of mechanical problem with complex geometry and three-dimensional stress repartition can be tackled by means of 3D numerical simulation to take into account the different hypotheses. Consequently, a numerical analysis is aimed to simulate this contact condition on the ball screw system. As for any numerical simulation, the modelling has to be validated in light of experimental data. Hence, the aim of this project is to study the load distribution on the ball screw system to improve the numerical simulation.

Because of complexity of the geometry, the project is conducted on several steps to establish a numerical model able to reproduce the real load distribution. The first one was the validation of a simplified 2D numerical model of the ball screw system in light of 2D experimental data [2]. On a second step, presented in this paper, a 3D numerical simulation has to be validated on a simplified 3D ball thrust bearing geometry. Finally, the last step will be to validate a 3D numerical simulation on the real geometry of the ball screw system.

Several experimental methods allow 3D measurements through a whole solid like Digital Volume Correlation coupled with tomography devices [3] to measure kinematics data or Scattered Light Photoelasticity (SLP) [4-6] to determine 3D stress repartition. For that, in this work, SLP 
method has been employed in order to study pressure distribution in the simplified 3D ball thrust bearing system.

In this paper, the SLP technique and the numerical simulation are firstly described. Then, a comparison between both is performed to see whether the modelling can be validated in light of the experimental data.

\section{Scattered Light Photoelasticity (SLP)}

\subsection{SLP principle}

A way to study experimental stress by photoelasticity in 3D geometries is to perform stress freezing technique coupled with mechanical slicing. Each plane slice is analysed with a classical 2D polariscope [7]. However, this process is destructive and does not enable to obtain 3D stress repartition on a same specimen. Hence, to avoid these difficulties, optical non-destructive techniques have been developed dealing with the polarization of scattered light in a birefringent material [8-11].

Based on these non-destructive techniques, the team Photomechanics and Experimental Mechanics of the PPRIME Institute of Poitiers developed SLP method using numerical images of scattered light through the studied material. This method consists in isolating a slice in the studied photoelastic material between two plane laser beams [4]. In the experimental device, a monochromatic laser source is first separated in two laser beams, each one going firstly in a convergent lens and secondly into a cylindrical lens so as to have two plane laser beams (figure 3). Once they isolate a slice by illuminating two sections of the studied specimen. The scattered light of each section corresponds to a speckle pattern due to interferences of light beams. These interferences depend on the birefringent properties of the material in the isolated slice.

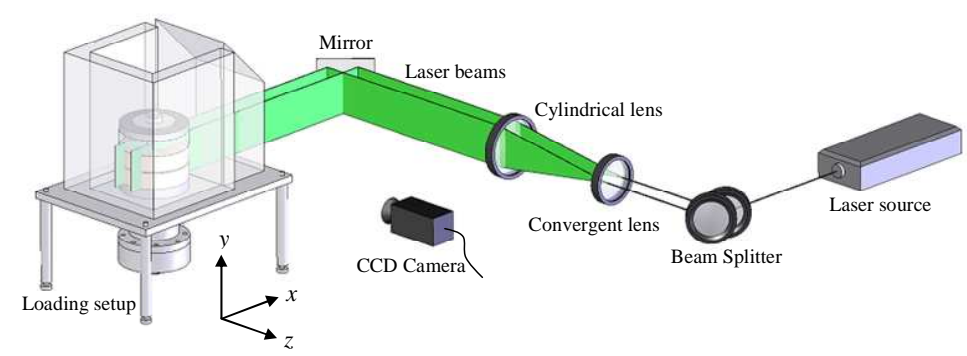

Fig. 3. SLP experimental setup

To acquire data in the isolated slice, three images are recorded by CCD camera: the first one corresponds to the diffused light for the first section illuminated alone (the recorded intensity is named $I_{1}$ ), the second one for the second section illuminated alone $\left(I_{2}\right)$, and the last one with both planar laser beam illuminating both sections at the same time $(I)$. The diffused light intensity can be separated in two parts, one corresponding to the background intensities $\left(I_{F}\right)$ and one corresponding to the laser speckle intensities $\left(I_{g}\right)$.

From $[4,12]$, recorded intensities can be expressed by:

$$
\left\{\begin{array}{l}
I_{1}=I_{1 F}+I_{1 g} \\
I_{2}=I_{2 F}+I_{2 g} \\
I=I_{1}+I_{2}+2 \sqrt{I_{1 g}} \sqrt{I_{2 g}} \gamma \cos \left(\psi_{1}+\psi_{2}+\eta\right)
\end{array}\right.
$$


in which $\psi_{1}$ and $\psi_{2}$ are the random phases of the speckle fields, $\eta$ is a function of optical characteristics of the slice, and $\gamma$ the correlation factor of both speckle fields given by [13] for small slice:

$$
\gamma^{2}=1-\sin ^{2}(2 \alpha) \sin ^{2}\left(\frac{\varphi}{2}\right)
$$

This expression contains mechanical parameters $\alpha$ et $\varphi$ (respectively isochrome and isoclinic parameters), identical to the ones obtained in $2 \mathrm{D}$ photoelasticity. By assuming that the birefringent state is constant along the slice and is described by a $2 \mathrm{D}$ optical index tensor, isochrome parameter $\varphi$ is given by:

$$
\varphi=\frac{2 \pi e}{\lambda}\left(n_{1}-n_{2}\right)
$$

and the isoclinic parameter $\alpha$ function of the stress tensor components (with $(x, y)$ defined by the plane laser beams):

$$
\alpha=\frac{1}{2} \arctan \left(\frac{2 \sigma_{x y}}{\sigma_{x x}-\sigma_{y y}}\right)
$$

where $e$ is the thickness of the slice and $\lambda$ the wavelength of the light. The difference of optical indexes $\left(n_{1}-n_{2}\right)$ is linked to the secondary principal stress difference $\left(\sigma_{1}-\sigma_{2}\right)$ by Maxwell's formulation [8]:

$$
\left(n_{1}-n_{2}\right)=C\left(\sigma_{1}-\sigma_{2}\right)
$$

$C$ is the photoelastic constant of the material, as well as mechanical characteristics $(E, v)$, are determined experimentally by mark tracking technique [5,14].

The photoelastic fringes cannot be observed directly with the intensity $I$ because the scattered intensity is a speckle field and $I_{I g}, I_{2 g}, \psi_{l}$ and $\psi_{2}$ are random values. Consequently, to determine the correlation factor and the fringes, a statistical analysis is performed on the speckle pattern $[4,12]$. By calculating averages (noted $<>$ ) and variances (var) of intensity fields, the correlation factor is determined by the following formulation:

$$
k^{2} \gamma^{2}=\frac{\operatorname{var}^{2}-\operatorname{var}_{1}^{2}-\operatorname{var}_{2}^{2}}{2\left\langle I_{1}\right\rangle\left\langle I_{2}\right\rangle}
$$

where $k$ is a factor related to scattered light properties of the material.

For these SLP experiments, specimens are usually casted in epoxy resin with the addition of small silica powder in order to induce scattered light phenomenon.

\subsection{Application of SLP on a 3D ball thrust bearing}

To be able to use SLP technique, a demonstrator ball thrust bearing system has been manufactured in casted epoxy resin (figure 4). Silica powder has been included during the cast in order to increase the scattering phenomenon. This powder represents $0.05 \%$ of the total mass of the specimen. The mechanical characteristics and the photoelastic constant of the material were measured by the 
experimental process shown on figure 3 performed on a normalised tensile test specimen casted at the same time as the ball thrust bearing.

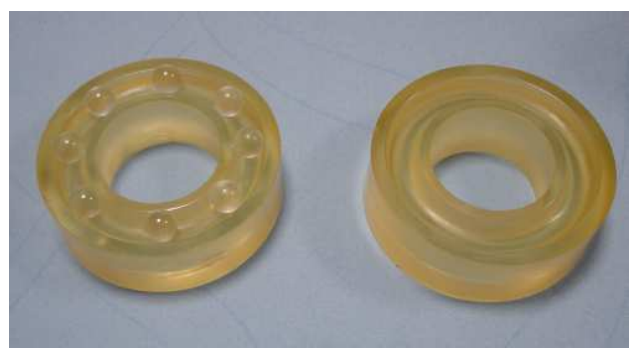

Fig. 4. Ball thrust bearing system made in epoxy

A device enables to apply a load on the ball thrust bearing specimen with an hydraulic jack. Four PMMA thick plates create a tank where a liquid having a similar optical index to the one of the epoxy resin is added. The liquid immerses the ball thrust bearing to avoid any reflection and refraction phenomenon on surfaces of the specimen. The balls introduced in the system are made of PMMA which is transparent and has a mechanical behaviour similar to the epoxy one. The optical slice used for this study has a thickness of $5 \mathrm{~mm}$ and is located at the medium plane of the observed ball. The comparison with FE model is performed on isochromatic fringes as they are directly linked to the principal stress differences by equations (4) and (6).

\section{Numerical simulation and fringe calculation}

The numerical simulation has been performed on the FEM commercial software Abaqus. Because of load distribution and of geometrical symmetries, only 1/8 of the full specimen is modelled (figure 5). The load is applied on the top surface and is controlled by a displacement condition. On the bottom surface, a zero-displacement condition is imposed. Mesh is refined at the vicinity of the contact zone in order to have more accurate results in this zone of interest. The considered material behaviour in this numerical simulation is the one obtained experimentally by the process presented in figure 3 for the epoxy resin.

As shown on figure 5, the part corresponding to the experimental slice is isolated to plot the isochromatic fringes in order to be compared with the experimental ones. Indeed, the isochromatic parameter $\varphi$ can be calculated from equations (4) and (6) with the extracted principal stresses. Nevertheless, the chosen numerical slice thickness is too high to assume a plane state for stresses, the stress field may change along the thickness because of the possible three-dimensional specimen behaviour. To avoid this problem, the local model of the slice is discretized in thin sub-slices of nodes where the stress field can be assumed to remain constant in their thickness [15]. The parameters $\varphi$ and $\alpha$ can be calculated from all nodes of each sub-slice stress field components $\sigma_{x x}, \sigma_{y y}$ and $\sigma_{x y}$.

Figure 5 shows the results for isochromatic fringes obtained numerically for a specific sub-slice to be compared to the experimental ones. 


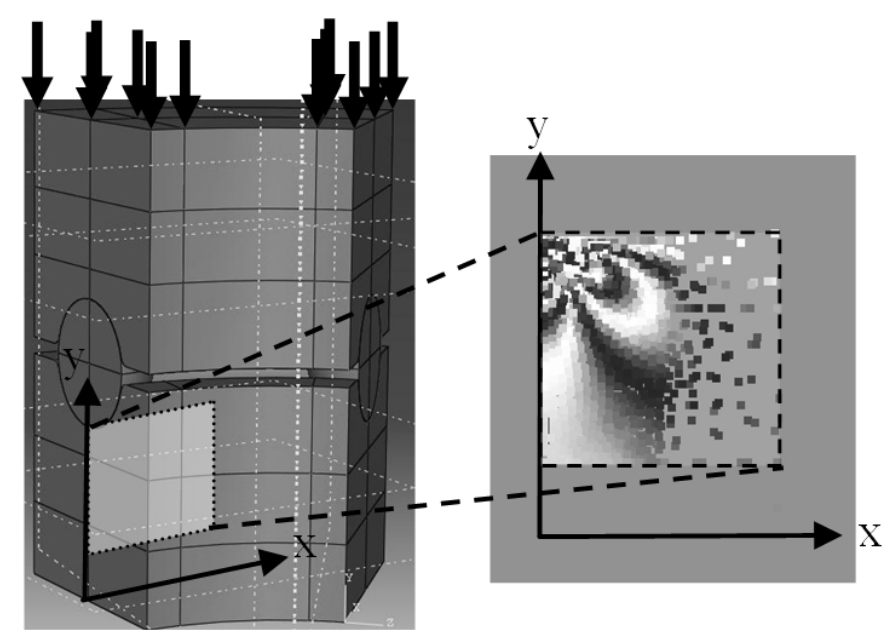

Fig. 5. Numerical simulation of $1 / 8$ of the specimen and results obtained on the sub-slice corresponding to the medium plane of the contact zone

\section{Comparison between numerical simulation and SLP results}

Figure 6 shows the experimental fringes that can be compared to the one of the numerical simulation previously presented in figure 5 for an applied load of $1000 \mathrm{daN}$.

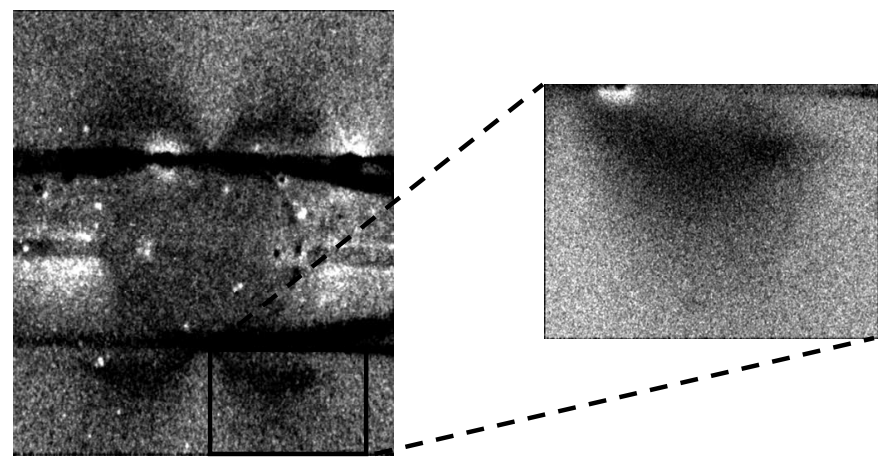

Fig. 6. Experimental results for an applied load of $1000 \mathrm{daN}$ and a slice of $5 \mathrm{~mm}$

Results are obviously not the same between numerical and experimental approaches, which means that this numerical model and its boundary conditions cannot be validated in light of these experimental results. Different loads were then tested experimentally to see the evolution of fringes at the contact zone (figure 7). Number of fringes did not increase with the load whereas their positioning around the ball varies with the $X$ axis. This result indicates a staggering of the contact zone with the load, which increases the surface for the stress distribution. This result is also different from the one obtained numerically for the same loads where number of fringes increased with the load but not the contact area that remains almost the same in spite of the load increase. This observation indicates most probably that the behaviour law of the ball is different between both approaches. The next step is to evaluate the real behaviour of these balls, or else to work with balls on which we can know perfectly the mechanical characteristics to perform another numerical calculation. 


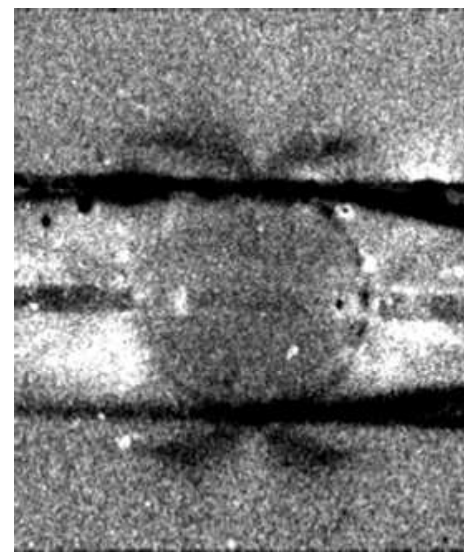

(a)

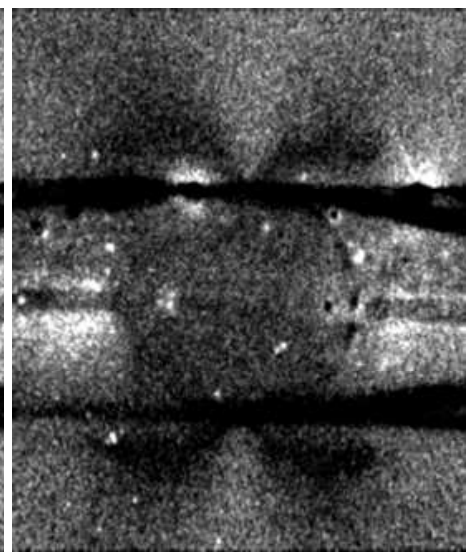

(b)

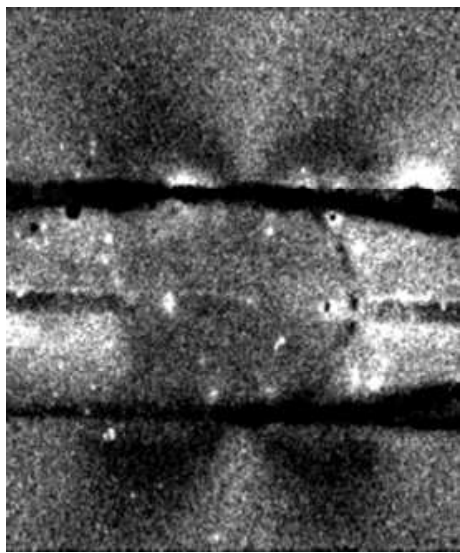

(c)

Fig. 7. Experimental results for an applied load of (a) $250 \mathrm{daN}$, (b) $1000 \mathrm{daN}$ and (c) $1750 \mathrm{daN}$. Staggering of the contact zone with the load.

For a global analysis of the system, comparison on imposed displacement versus loading also gives differences between both approaches. The displacement of the surface on which the load is applied experimentally was measured by mark tracking technique [14]. By this global analysis on the differences between the experimental displacement and the numerical imposed displacement, loading conditions and boundary conditions in the numerical simulation appear to be not perfectly relevant of the experiment and are currently under modifications. Hence, the global analysis gives the same conclusion than local study.

\section{Conclusion}

In this paper, two three-dimensional approaches are presented, one based on numerical simulation and one based on experiment. In this project, the numerical simulation had to be assessed by the experiment to be relevant of the pressure distribution on contacts in a ball-screw system. Numerical simulation made for this demonstrator ball-screw system could not be assessed by SLP threedimensional measurement technique because of differences in results between both approaches.

These differences are observed for both local fringes and global displacements, which indicate that numerical simulation and assumptions do not match the experimental conditions on the epoxy casted specimen. The numerical modelling is currently under modifications in order to be able to validate this geometry before starting the third step of the study on a ball-screw system geometry closer to the real one.

\section{References}

1. D.A. Hills, D. Nowell, A. Sackfield, Mechanics of Elastic Contacts (Butterworth Heinmann, 1993)

2. R. Bertolaso, Y. Barranger, M. Cheikh, J.C. Dupré, A. Germaneau, P. Doumalin, ICSAAM 2009 (Tarbes, 2009, France)

3. A. Germaneau, P. Doumalin, J.C. Dupré, NDT\&E Int 41, 407-415 (2008)

4. J.C. Dupré, A. Lagarde, Expl Mech 37(4), 393-397 (1997)

5. A. Germaneau, P. Doumalin, J.C. Dupré, ICEM13 (Alexandropolis, 2007, Greece)

6. A. Germaneau, F. Peyruseigt, S. Mistou, O. Dalverny, P. Doumalin, J.C. Dupré, Journal of Engineering Tribology 222, 647-656 (2008) 
7. R.D. Peindl, M.E. Harrow, P.M. Connor, D.M. Banks, D.F. D’Alessandro., Experimental Mechanics 44(3), 228-234 (2004)

8. A. Lagarde, P. Oheix, J. Brillaud, Mechanics Reserach Communications 3, 107-112 (1976)

9. S.Y. Keshavan, W. Weber, Mechanics Reserach Communications 4(6), 381-387 (1977)

10. H. Aben, Integrated photoelasticity (McGraw-Hill, 1979)

11. R. Desailly, A. Lagarde, Revue Française de Mécanique, 47-55 (1984)

12. N. Plouzennec, J.C. Dupré, A. Lagarde, Symposium I.U.T.A.M. (Poitiers, 1998, France)

13. R. Desailly, A. Lagarde, Journal de Mécanique Appliquée 4(1), 3-30 (1980)

14. N. Bretagne, V. Valle, J.C. Dupré, NDT\&E Int 38, 290-298 (2005)

15. A. Zenina, J.C. Dupré, A. Lagarde, Symposium I.U.T.A.M. (Poitiers, 1998, France) 